\title{
EPILEPSIA Y EMBARAZO
}

Eduardo Palacios MD*, Karen Cárdenas MD**

\section{Resumen}

La epilepsia es la entidad neurológica que con mayor frecuencia requiere tratamiento continuo durante la gestación. Se revisan las posibles complicaciones en la madre, en que forma puede afectarse el feto y la probabilidad teratogénica de los anticonvulsivantes. Es importante programar el embarazo para elegir el medicamento adecuado siendo la época libre de crisis la más conveniente. El médico debe buscar ante todo controlar las crisis durante el embarazo y buscar que el fármaco sea el menos teratogénico, pues casi todos los anticonvulsivantes tienen efectos nocivos para el feto. El ácido valproico continúa siendo el de mayor riesgo para producir malformaciones congénitas y trastornos cognitivos detectables en la niñez. Otros medicamentos como levetiracetam, lamotrigina, carbamazepina o fenitoina han demostrado ser menos tóxicos. Debe evitarse la politerapia y administrar ácido fólico para favorecer la normalidad del embarazo y su producto.

Palabras clave: embarazo, epilepsia, anticonvulsivantes, teratogenicidad.

\section{EPILEPSY AND PREGNANCY}

\section{Abstract}

Epilepsy is the most common neurologic condition requiring continuing treatment during pregnancy. This paper reviews the possible complications for the mother, how may the fetus be affected and the possible teratogenicity of anticonvulsants. Conception must be planned in order to choose the proper medication and a seizure-free period is the most convenient time. Proper seizure control and choosing the least teratogenic agent is the primary goal for almost all anticonvulsants induce harmful effects on the fetus. Valproic acid carries the highest risk associated with congenital malformations and cognitive impairment detectable during childhood. Other anticonvulsants such as levetiracetam, lamotrigine, carbamazepine or phenytoin have been found to be less toxic. Polytherapy must be avoided and folic acid should be administered to have a favorable gestational and newborn outcome.

Key words: Pregnancy, epilepsy, anticonvulsants, teratogenicity

Fecha recibido: julio 13 de 2015 - Fecha aceptado: julio 29 de 2015

* Jefe del Servicio de Neurología, Hospital de San José. Miembro de la Sociedad de Cirugía de Bogotá. Profesor Titular, Fundación Universitaria de Ciencias de la Salud. Bogotá DC, Colombia.
** Residente III de Neurología. Fundación Universitaria de Ciencias de la Salud. Bogotá DC, Colombia. 


\section{Introducción}

El propósito de esta publicación es revisar la conducta que se debe adoptar con la paciente epiléptica durante el embarazo, ver de qué manera esta afección puede afectar a la mujer o al feto y hacer énfasis en un problema conocido desde hace varios años pero que continúa siendo la gran preocupación del médico y de la embarazada, los efectos teratogénicos de los medicamentos anticonvulsivantes.

Buscamos responder las preguntas que con frecuencia hacen las pacientes a sus médicos; cuáles son las recomendaciones a la enferma epiléptica que desea un embarazo; cuál la conducta de la que se embaraza sin tener en cuenta los riesgos teratogénicos de los medicamentos que viene recibiendo; cuáles son los anticonvulsivantes que debe recibir en caso de embarazo y cuáles los riesgos para la madre o el feto en caso de convulsiones.

\section{Epilepsia en mujeres en edad fértil}

Vale la pena anotar que las epilépticas se embarazan con menor frecuencia al compararlas con mujeres sanas de su misma edad. ${ }^{1-3}$ Puede ser por temor a transmitir la enfermedad a sus hijos o por conocer los problemas teratogénicos de los medicamentos que reciben. Algunos estudios realizados en el Reino Unido informan que el $33 \%$ de las epilépticas no desean embarazarse por miedo a su enfermedad, por heredarle el problema a los hijos y por la posibilidad de defectos congénitos. El $90 \%$ tienen un embarazo normal, no obstante el temor a problemas congénitos en el recién nacido es justificado. ${ }^{4-6}$ En diversas épocas de la vida la mujer presenta cambios importantes en su sistema hormonal, bien evidentes durante el embarazo lo que en una u otra forma puede comprometerla o al producto. Las hormonas sexuales femeninas pueden producir cambios en la excitabilidad de las neuronas, los estrógenos se comportan como excitatorios y la progesterona como inhibitoria. ${ }^{5}$

\section{Epilepsia catamenial}

Se ha dado este nombre a las crisis que se presentan o se incrementan durante el ciclo menstrual, más frecuentes en los días previos o durante la menstruación. Se ha relacionado en especial con el aumento de estrógenos aunque no hay muchos estudios al respecto; se considera que por lo menos el $30 \%$ de las epilépticas presentan más crisis durante el ciclo menstrual ${ }^{7}$ sobre todo si son de origen focal. Se han propuesto varios tratamientos para este tipo de crisis con dieta hiposódica en los días anteriores a la menstruación o la administración de acetazolamida, 250 a 500 mg diarios comenzando siete días antes hasta el fin del periodo o una benzodiacepina de preferencia clobazam, 5 a $10 \mathrm{mg}$ en la noche por el mismo tiempo que la anterior. El diurético puede producir parestesias y el clobazam somnolencia. ${ }^{7}$

\section{Hormonas y epilepsia}

Hemos comentado la importancia de los cambios hormonales en la incidencia de las crisis epilépticas. $\mathrm{Al}$ inicio de la menopausia los estrógenos que se han considerado facilitatorios de las crisis pueden aumentar sus niveles, por el contrario la progesterona que se considera antiexcitatoria disminuye, cambios que favorecen un incremento de los episodios convulsivos en esta época. Cuando la paciente está ya en menopausia las crisis disminuyen. ${ }^{8}$ Tanto la epilepsia como los medicamentos pueden producir alteraciones hormonales que dificultan el embarazo ${ }^{2,4}$ y son más frecuentes las malformaciones fetales en las que reciben tres anticonvulsivantes. ${ }^{8-10}$

\section{Epilepsia y embarazo}

Lo ideal es que la embarazada no reciba medicamentos, muchas solicitan que no se les administre o la suspenden en forma voluntaria, pero esto no es tan sencillo. ${ }^{11,12}$ Hay diversos estudios que muestran cómo las crisis durante el embarazo conllevan a problemas fetales así, durante crisis generalizadas en eclampsia hay sufrimiento fetal, en especial bradicardia severa seguida de taquicardia compensatoria, lo que segura- 
mente produce isquemia en el feto. ${ }^{11,13}$ También se ha observado vasoconstricción de la circulación placentaria, lo que se traduce en isquemia con repercusiones graves para el feto. ${ }^{11,14}$

Las crisis generalizadas tónico clónicas suelen cursar con acidosis e hipoxia con posible repercusión en el feto. ${ }^{15}$ En el curso del embarazo la mujer experimenta cambios importantes hormonales lo mismo que aumento de peso, lo que facilita las crisis y exige cuidado en la dosis del medicamento. ${ }^{5,10}$ Lo ideal sería no tomar fármacos durante el embarazo; pero si se considera necesario lo importante es recomendar los más adecuados y administrarlos en dosis mínimas útiles. ${ }^{12,15,16}$

Hay diferentes opiniones entre los autores en relación con el incremento o no de las crisis durante la gestación. En general se considera que no se modifican. ${ }^{11,17}$ En la epiléptica hay ciertos riesgos que parecen propios del embarazo, por ejemplo se ve mayor mortalidad que llega a ser hasta diez veces más que en grupos comparativos de mujeres sanas. Los hijos de estas madres son más pequeños y es interesante anotar que inclusive se observa en aquellos de madres epilépticas que no toman anticonvulsivante durante el embarazo. ${ }^{11,17,18}$ Con los comentarios anteriores hemos querido mostrar cómo el embarazo en la epiléptica puede llegar a convertirse en un dilema para la paciente y en un problema para el médico que debe instruirla y aconsejarla. ¿Es conveniente embarazarse? Y abordar el siguiente punto que se relaciona con la administración de medicamentos.

\section{Efectos teratogénicos de los anticonvulsivantes}

Se considera que pueden producir malformaciones mayores, menores o las dos ${ }^{15,18-21}$ (Tabla 1). Las primeras son las que causan defectos anatómicos que interfieren con la normal función de un órgano o que pueden llegar a comprometer la vida; por ejemplo malformaciones cardiacas, espina bífida, paladar hendido, labio leporino o microcefalia. Pueden ocurrir hasta tres veces más que en hijos de madres sanas que varían entre 3 y el 9\%. Las segundas son aquellas que no comprometen la vida del paciente y por lo general solo causan pro-

\begin{tabular}{|l|l|}
\multicolumn{2}{c}{$\begin{array}{c}\text { Tabla I. Efectos teratogénicos de algunos } \\
\text { medicamentos anticonvulsivantes }\end{array}$} \\
\hline $\begin{array}{c}\text { Medicamentos } \\
\text { anticonvulsivantes } \\
\text { (monoterapia) }\end{array}$ & \multicolumn{1}{c|}{$\begin{array}{c}\text { Efectos teratogénicos } \\
\text { asociados }\end{array}$} \\
\hline Ácido valproico & $\begin{array}{l}\text { Espina bífida, paladar hendido, } \\
\text { hipospadias, malformaciones } \\
\text { cardiacas, polidactilia, } \\
\text { craneosinostosis, IQ bajo, autismo }\end{array}$ \\
\hline Fenobarbital & \begin{tabular}{l} 
Cardiovasculares \\
\hline Topiramato
\end{tabular} \\
\hline Fenitoína & $\begin{array}{l}\text { Pabio leporino, paladar hendido hendido, labio leporino, } \\
\text { malformaciones cardiovasculares }\end{array}$ \\
\hline Carbamazepina & Espina bífida \\
\hline
\end{tabular}

blemas de tipo estético como el dismorfismo cráneo facial, hipertelorismo y malformaciones de las uñas. Se considera que estas llegan a ser también hasta tres veces más frecuentes en los hijos de madres epilépticas que en madres sanas..$^{14,15,18,22}$ En los últimos años se ha dado especial importancia a los problemas cognitivos que pueden presentar hijos de madres epilépticas y que aparecerán durante el desarrollo, por ejemplo hiperactividad, bajo coeficiente intelectual, dificultad en el aprendizaje y autismo..$^{21,23-25}$

Estudios a gran escala realizados por la Academia Americana de Neurología, la Asociación Americana de Epilepsia y el Registro Internacional de Medicamentos Anticonvulsivantes durante el Embarazo (EURAP study group) ${ }^{10,15,17,26-30}$ llegan a las siguientes conclusiones: las malformaciones se observan con mayor frecuencia si el medicamento se administra en el primer trimestre del embarazo, cuando la dosis es alta hay mayor posibilidad de que ocurran, tomar un medicamento durante el embarazo es menos riesgoso pero tres aumentan hasta tres veces la posibilidad.

Es claro que el anticonvulsivante con mayores problemas teratogénicos es el ácido valproico y en especial si se emplea en dosis mayores a $700 \mathrm{mg}^{11,20,25}$, puede producir malformaciones mayores hasta en $10 \%$ de los hijos, en especial espina bífida, paladar hendido, hipospadias, malformaciones cardiacas, polidactilia y craneosinostosis. ${ }^{27,30-32} \mathrm{Si}$ la dosis llega a $1500 \mathrm{mg}$ diarios la posibilidad es de $20 \%{ }^{6,27}$ Se ha descrito un 
conjunto de características fenotípicas en hijos de madres que recibieron valproato, las cuales no se observan con otros anticonvulsivantes, consistentes en pliegue epicántico, estrabismo, puente nasal plano, surco nasolabial poco profundo y labio superior pequeño, que en conjunto conforman el llamado síndrome fetal por valproato. ${ }^{21,33-35}$ Algunos estudios informan una relación importante entre dismorfismo facial e inteligencia verbal con otros antiepilépticos durante el embarazo. ${ }^{24,34,36,37}$

En casos de politerapia cuando se incluye el valproato, aumentan las posibilidades de malformaciones. ${ }^{6,10,13-14}$ A la edad de seis a ocho años se ha encontrado hasta diez puntos menos en el coeficiente intelectual de niños cuyas madres lo recibieron en el primer trimestre del embarazo. ${ }^{21,23,35-38} \mathrm{El}$ empleo de valproato en dosis útiles durante el primer trimestre del embarazo se ha asociado también a autismo. ${ }^{37-41}$ Cuando la lamotrigina y el valproico se administran al tiempo puede llegar al $12 \%$ de malformaciones. ${ }^{11,19,21,22}$ Con el fenobarbital se encuentran problemas similares predominando las malformaciones cardiovasculares ${ }^{42-44}$; es un medicamento que se emplea cada vez menos en nuestro medio; no es recomendable en el embarazo. ${ }^{11}$

Dos medicamentos que parecen confiables en la gestación son el levetiracetam y la lamotrigina ${ }^{10}$, pueden ser responsables de malformaciones menores (de las uñas y falanges distales) en porcentajes bajos en relación con su empleo. Algunas publicaciones informan una mínima posibilidad de espina bífida con carbamazepina $^{45}$, medicamento que también parece más o menos seguro. Se sugiere emplear las dosis mínimas terapéuticas útiles. Algunos autores han informado hasta $3.3 \%$ de malformaciones congénitas cuando las madres han recibido carbamazepina en el primer trimestre del embarazo. ${ }^{46,47}$

En un momento se consideró la lamotrigina como el fármaco más recomendado, pero se encontró que los niveles séricos disminuyen en forma importante durante la gestación, regresando rápidamente en el posparto a los niveles previos; es muy importante el monitoreo de estos niveles antes, durante y después de la gestación..$^{22,48}$
Según los diferentes estudios de los registros internacionales la lamotrigina aumenta el riesgo de malformaciones congénitas mayores entre 2.7 y $3.2 \%$; sin embargo existe controversia respecto a si la dosis está asociada o no. El registro del Reino Unido encuentra que a mayor dosis aumenta el riesgo con una significancia estadística limítrofe; dicha asociación no se ha demostrado en otros estudios como los registros americanos o australiano y se cree que esta diferencia se debe a los cambios en niveles séricos de lamotrigina durante la gestación y otros problemas de diseño de los estudios. ${ }^{49}$

Otro medicamento que se emplea con frecuencia es la fenitoína. En el recién nacido se puede encontrar paladar hendido, labio leporino, malformaciones cardiovasculares y urológicas hasta tres veces más que en mujeres sanas. Puede hallarse también el llamado síndrome fetal hidantoínico en el que suele verse hipoplasia de las falanges distales de los dedos, hipertelorismo, bajo peso al nacer y en ocasiones patología del paladar. ${ }^{11,50,51}$ Según algunas publicaciones estas malformaciones pueden llegar a comprometer hasta $11 \%$ de los hijos de mujeres tomando hidantoína; en la actualidad no se observa tan frecuente tal vez por el empleo de ácido fólico. Solo si se considera indispensable se continúa la fenitoína durante el embarazo. ${ }^{11}$

El levetiracetam es uno de los más empleados en epilepsia por sus buenos resultados y poca toxicidad. ${ }^{52,53}$ En varios estudios se ha demostrado bajo riesgo de malformaciones congénitas cuando se emplea en monoterapia, por lo que su uso se recomienda durante el embarazo. ${ }^{11,52}$ La única malformación importante observada en menos de $1 \%$ de los hijos de madres epilépticas que recibieron este medicamento es hernia inguinal. ${ }^{11,51} \mathrm{El}$ empleo en politerapia tiene riesgo de malformaciones congénitas mayores, en especial cuando se asocia con valproato o carbamazepina. El riesgo es menor con lamotrigina. ${ }^{53,54}$

El topiramato, medicamento de empleo frecuente en migraña y epilepsia, causó gran impacto ya que sus resultados parecían muy alentadores con poca tóxicidad. Se emplea con buenos resultados en estas dos entidades pero se considera teratogénico, hay posibilidades de 
paladar hendido y labio leporino en cerca de $2 \% .^{51,55}$ La posibilidad de malformaciones en el paladar es diez veces mayor que en mujeres que no toman medicamentos antiepilépticos ${ }^{56} \mathrm{Al}$ principio fue catalogado como riesgo $\mathrm{C}$ durante el embarazo pero hoy es $\mathrm{D}$. Tampoco es recomendable en el embarazo. ${ }^{11,57}$

La carbamazepina se ha considerado relativamente segura durante el embarazo, la mayoría de las complicaciones se consideran menores. Con menos de 400 mg por día las malformaciones no llegan a $2 \%$; si la dosis es $\geq 1$ las malformaciones congénitas mayores llegan a $7 \% .^{6}$ Tal vez la más temida es la espina bífida que se ve en $2 \%{ }^{47}$, es decir $80 \%$ menos que con el valproato y más o menos similar a la observada con otros anticonvulsivantes como lamotrigina, fenobarbital, levetiracetam. ${ }^{58}$

En general los estudios se han realizado durante el primer trimestre del embarazo. ${ }^{50-51,58}$ Más difícil resulta la evaluación cognitiva de estos niños ya que las investigaciones son a más largo plazo..$^{25,59,60} \mathrm{Al}$ comienzo se realizaron en aquellos con malformaciones menores cuyas madres recibían anticonvulsivantes, en la actualidad se estudian a largo plazo los de madres epilépticas donde no todos cursan con malformaciones. De nuevo el ácido valproico es el más comprometido, a los cuatro años de edad el coeficiente intelectual es hasta nueve puntos menor que con lamotrigina y diez menos que en hijos de madres que no han recibido medicamentos. ${ }^{25-26,61}$

En la actualidad se realizan varios estudios prospectivos para definir esta posible patología. Otra observación importante es la relación entre ingesta de ácido valproico durante el embarazo y presencia de autismo y trastornos de hiperactividad en cerca de $7 \%$ de estos niños contra $1 \%$ de la población general. ${ }^{40,51}$ El estudio EURAP señala que hay una relación importante entre la teratogénesis de los medicamentos anticonvulsivantes y los antecedentes familiares de malformaciones congénitas. ${ }^{21}$

Investigaciones realizadas en el Reino Unido y Estados Unidos demostraron que los pacientes expuestos a ácido valproico en monoterapia tienen menor co- eficiente intelectual comparado con carbamazepina, lamotrigina o fenitoina; también se encontraron trastornos de memoria y habilidad verbal. A mayor dosis de valproico menor coeficiente intelectual. ${ }^{26,30,61} \mathrm{El}$ estudio NEAD sugiere que la exposición a medicamentos anticonvulsivantes lleva a cambios en la lateralización cerebral con predominio de zurdos. ${ }^{62}$ Riesgo mayor de malformaciones: ácido valproico-topiramato-fenobarbital. ${ }^{11,12,14}$ Probablemente seguras: levetiracetam, lamotrigina y caramazepinafenitoína. ${ }^{11,12,14}$

Con otros medicamentos de empleo común en epilepsia no hay estudios suficientes para dar una opinión como son oxcarbazepina, lacosamida y gabapentin. Cabe anotar que suele ocurrir disminución de niveles de fármacos antiepilépticos como carbamazepina, lamotrigina y fenitoina, por lo que es importante el monitoreo de cifras séricas durante el embarazo. ${ }^{11,12,15}$

En este periodo se aconsejan algunas conductas específicas para prevenir las crisis convulsivas (Tabla 2), las cuales se discuten a continuación. La administración de ácido fólico ${ }^{10,30,63-65}$ se recomienda desde hace varios años para prevenir los problemas teratogénicos de los medicamentos, se comienza a administrar antes del embarazo en dosis máxima de $4 \mathrm{mg}$ diarios, lo cual es suficiente para prevenir el riesgo de malformaciones congénitas. En varios productos alimentarios se ha agregado ácido fólico con el fin de obtener este beneficio. Aunque han aparecido algunos estudios que lo ponen en duda ${ }^{6,66}$, las recomendaciones del Colegio Americano de Ginecología y Obstetricia (2003) han prevalecido desde entonces. ${ }^{67}$

Una vez finalizado el embarazo se debe tener en cuenta la lactancia materna, pues la evidencia sugiere que el levetiracetam y la primidona pasan en cantidad importante a la leche materna, mientras que con ácido valproico, fenobarbital, fenitoina y carbamazepina es menor. ${ }^{63}$

Durante el embarazo y el parto pueden encontrase algunas complicaciones. En quienes reciben anticonvulsivante aumenta la posibilidad de parto antes de las 34 semanas, mientras en la epiléptica sin medicación no hay variación en relación con mujeres sanas. La 
Tabla 2. Medidas para tener en cuenta antes, durante y después de la gestación

\begin{tabular}{|c|c|}
\hline Medida & Conducta \\
\hline Suplemento de ácido fólico & Dosis 0.4 - $4 \mathrm{mg}$ día \\
\hline $\begin{array}{l}\text { No siempre es más seguro } \\
\text { cambiar o suspender de } \\
\text { manera abrupta el medica- } \\
\text { mento que venía tomando }\end{array}$ & $\begin{array}{l}\text { Llevar a la paciente a monoterapia } \\
\text { y a la dosis mínima efectiva }\end{array}$ \\
\hline $\begin{array}{l}\text { Monitorear niveles del me- } \\
\text { dicamento durante la ges- } \\
\text { tación }\end{array}$ & $\begin{array}{l}\text { Especialmente la lamotrigina } \\
\text { cambia sus niveles séricos duran- } \\
\text { te la gestación }\end{array}$ \\
\hline Tamizaje prenatal & $\begin{array}{l}\left(1^{\circ}, 2^{\circ} \text { y } 3^{\text {er }} \text { trimestre }\right) \text { para de- } \\
\text { tectar defectos del tubo neural }\end{array}$ \\
\hline Vitamina K & $\begin{array}{l}\text { I0 mg oral a la madre el último } \\
\text { mes del embarazo, I mg intramus- } \\
\text { cular al recién nacido }\end{array}$ \\
\hline \multirow[t]{2}{*}{ Posparto } & $\begin{array}{l}\text { Seguimiento estricto de niveles } \\
\text { séricos del MAC }\end{array}$ \\
\hline & $\begin{array}{l}\text { Precauciones con la madre y el } \\
\text { recién nacido ya que durante el } \\
\text { puerperio puede aumentar la fre- } \\
\text { cuencia ictal (ej. baño del recién } \\
\text { nacido siempre con acompañante). }\end{array}$ \\
\hline
\end{tabular}

hiperemesis gravídica, el sangrado placentario y la hipertensión gestacional son algo más común en epilépticas con tratamiento, con una cifra de 1.5 a $2 \%$. No obstante el porcentaje de complicaciones obstétricas es muy bajo. ${ }^{11,17}$

La Tabla 3 presenta las complicaciones que puede producir la epilepsia durante el parto. Se han informado episodios convulsivos durante el parto y en el mes siguiente hasta en 3\%, si el episodio ocurre durante el parto se puede controlar fácil con benzodiacepinas. En general las crisis tienden a ser más frecuentes durante el último trimestre del embarazo, tal vez por el aumento de peso que implica elevar la dosis del medicamento y la abundancia de estrógenos que son muy convulsivógenos. ${ }^{17,21}$

La complicación más temida es el estado epiléptico, crisis tónico clónico generalizadas a repetición, que se presenta en menos de $2 \%$ de las pacientes y por lo general se debe a administración en dosis no terapéuticas o a suspensión abrupta de los mismos. Los informes
Tabla 3. Medidas recomendadas en el parto

\begin{tabular}{|l|l|}
\hline Vía del parto & $\begin{array}{l}\text { Puede ser llevada a parto vaginal a } \\
\text { menos que otra condición lo impida } \\
\text { Evite meperidina como analgésico } \\
\text { (disminuye umbral convulsivo) }\end{array}$ \\
\hline Crisis convulsiva & $\begin{array}{l}\text { Usar benzodiacepinas en dosis bajas } \\
\text { como primera opción, monitoreo de la } \\
\text { madre y recién nacido (depresión respi- } \\
\text { ratoria) }\end{array}$ \\
\hline $\begin{array}{l}\text { Otra crisis a pesar } \\
\text { de manejo inicial con } \\
\text { benzodiazepinas }\end{array}$ & $\begin{array}{l}\text { Levetiracetam, fenitoína o ácido val- } \\
\text { proico endovenosos }\end{array}$ \\
\hline Estado epiléptico & $\begin{array}{l}\text { Cesárea urgente, de monitoreo estricto } \\
\text { de la madre y el recién nacido, } \\
\text { tratamiento especializado }\end{array}$ \\
\hline
\end{tabular}

de la literatura no muestran variación en las secuelas en relación con otras pacientes. El tratamiento es igual al convencional para este tipo de patología con base en benzodiacepinas y levetiracetam o en su defecto fenitoína. ${ }^{27,30}$

\section{Conclusiones}

En resumen todos los anticonvulsivantes son en mayor o menor grado teratogénicos; se recomiendan los que hemos mencionado como más seguros. Siempre administrar ácido fólico durante el embarazo. Si la paciente pide opinión antes de embarazarse, insistir en que nunca se debe suspender en forma abrupta la medicación, de preferencia administrar uno de los medicamentos considerados como más seguros e iniciar ácido fólico, informarle que si bien es cierto que el embarazo puede presentar algunos riesgos para la madre y el feto tales como parto prematuro, algunas complicaciones obstétricas y riesgo de teratogenicidad, el porcentaje de complicaciones es bajo.

\section{Referencias}

1. Viinikainen K, Heinonen S, Eriksson K, Kalviainen R. Fertility in women with active epilepsy. Neurology. 2007; 69(22):2107-8.

2. Schupf N, Ottman R. Likelihood of Pregnancy in Individuals with Idiopathic/Cryptogenic Epilepsy: Social and Biologic Influences. Epilepsia. 1994; 35(4):750-6.

3. O'Connor SE, Zupanc ML. Women and epilepsy. J Pediatr Pharmacol Ther. 2009; 14(4): 212-20.

4. Crawford P, Hudson S. Understanding the information needs of woman with epilepsy al diferent lifestages: results of the 'Ideal World' survey. Seizure. 2003; 12(7): 502-507. 
5. Harden CL Pennell PB. Neuroendocrine considerations in the treatment of : men and woman with epilepsy. Lancet Neurol. 2013; 12(1): 72-83.

6. Tomson T, Battino D, Bonizzoni E, Craig J, Lindhout D, Sabers A, et al. Dose-dependent risk of malformations with antiepileptic drugs: an analysis of data from the EURAP epilepsy and pregnancy registry. Lancet Neurol. 2011; 10(7):609-17.

7. Nariño D, Grillo MP. Epilepsia catamenial. Acta Neurol Colomb. 2005; 21(1): 25-33.

8. Harden CL, Koppel BS, Herzog AG, Nikolov, BG, Hauser WA. Seizure frequency is associated with age at menopause in women with epilepsy. Neurology. 2003; 61(4): 451-55.

9. Morell MJ. Reproductive and metabolic disorders in women with epilepsy. Epilepsia. 2003; 44(suppl 4):11-20.

10. Harden CL, Meador KJ, Pennel PB, Hauser WA, Gronseth GS et al. Practice Parameter update: Management issues for women with epilepsy-Focus on pregnancy (an evidence-based review): Teratogenesis and perinatal outcomes. Report of the Quality Standards Subcommittee and Therapeutics and Technology Assessment Subcommittee of the American Academy of Neurology and American Epilepsy Society. Neurology. 2009; 73(2): 133- 141

11. Harden CL. Pregnancy and epilepsy. Continuum (Minneap, Minn.). 2014; 20(1, Neurology of Pregnancy):60-79.

12. Walker SP, Permezel M, Berkovic SF. The manegement of epilepsy in pregnancy. BJOG. 2009; 116(6):758- 67.

13. Craig JJ, Hunt SJ. Treating women with juvenile myoclnic epilepsy. Pract Neurol. 2009; 9(5):268-77.

14. Noe KH, Pack AM. Women's issues and epilepsy. Continuum (Minneap, Minn.). 2010; 16 (3, epilepsy): 159-78.

15. Harden CL, Pennel PB, Koppel BS, Hovinga CA, Gidal B, Meador KJ, et Al Practice Parameter update: Management issues for women with epilepsy-Focus on pregnancy (an evidence-based review): Vitamin K, folic acid, blood levels, and breastfeeding. Report of the Quality Standards Subcommittee and Therapeutics and Technology Assessment Subcommittee of the American Academy of Neurology and American Epilepsy Society. Neurology. 2009; 73(2):142-149.

16. Artama M, Isojärvi JL, Raitanen J, Auvinen A. Bird rate among patiens with epilepsy: a nationwide population-based cohort study in Finland. Am. J. Epidemiol. 2004; 159(11):1057-63.

17. Harden CL, Hopp J. Ting TY, Pennell PB, French JA, Hauser WA et al. Practice Parameter update: Management issues for women with epilepsy-Focus on pregnancy (an evidence-based review): Obstetrical complications and change in seizure frequency. Report of the Quality Standards Subcommittee and Therapeutics and Technology Assessment Subcommittee of the American Academy of Neurology and American Epilepsy Society. Neurology. 2009; 73 (2):126-132.

18. Pennell PB. Pregnancy in the woman with epilepsy. Maternal and fetal outcomes. Semin Neurol. 2002; 22(3): 299-308.

19. Holmes LB, Mittendorf R, Shen A, Smith CR, Hernandez-Diaz S. Fetal effects of anticonvulsivant politherapies: different risks from different drugs combinations. Arch Neurol. 2011; 68(10): 1275-81

20. Morrow J, Russell A, Guthrie E, Parsons L, Robertson I, Waddell R et al. Malformations risks of antiepileptic drugs in pregnancy: a prospective study from the UK epilepsy and pregnancy register. J Neurol Neurosurg psychiatry 2006; 77(2): 193-8.

21. Tran TA, Leppik IE, Blesi K, Sathanandan ST, Remmel R. Lamotrigine clearance during pregnancy. Neurology. 2002;59(2):251-5.

22. Tran TA, Leppik IE, Blesi K, Sathanandan ST, Remmel R. Lamotrigene clearence during pregnancy. Neurology. 2002; 59(2): 251-255.

23. Vajda FJ, O’Brian TJ, Graham J, Lander CM, Eadie MJ. Associations between particular types of fetal malformation and antiepileptic drug exposure in utero. Acta Neurol Scand. 2013; 128(4): 228-234

24. Pennel PB. Antiepileptic drugs during pregnancy: What is known and wich ADEs seem to be safest? Epilepsia. 2008; 49 sup. 9 ):43-55.

25. Meador Kj, Baker GA ,Browning N, Cohen MJ, Bromley RL, Clayton-Smith $\mathbf{J}$ et al. Fetal antiepileptic drug exposure and cognitive outcomes at age 6 years
(NEAD study): a prospective observational study. Lancet Neurol. 2013; 12 (3):244-253.

26. Meador KJ, Loring DW. Risks of in utero exposure to valproate. JAMA. 2013; 309(16):1730-31

27. Klein AM. Epilepsy cases in pregnant and pospartum women: a practical approach. Semin Neurol. 2011; 31(4):392-396.

28. Vajda F, O'Brian T. Valproic acid use in pregnancy and congenital malformations. N Engl J Med. 2010; 363(18):1771; author reply 1771-2.

29. Jentink J, Loane MA, Dolk H, Barisic I, Garne E, Morris JK et al.. Valproic Acid monotherapy in pregnancy and major congenital malformations. N Engl J Med. 2010; 362(23): 285-293.

30. DiLiberti JH, Farndon PA, Dennis NR, Curry CJ. The fetal valproate syndrome. Am J Med Genet. 1984;19(3):473-81.

31. Martínez-Ferri M. Peña-Mayor P, López-Fraile L, Castro-Vilanova MD, Escartin A, Moro $\mathrm{M}$ et al. Malformaciones y muerte fetal en el registro español de fármacos antiepilépticos y embarazo: resultados a los 6 años. Neurología. 2009; 24(6): 360-365.

32. Wiszinsky DF, Nambisan M, Surve T, Alsdorf RM, Smith CR, Holmes LB. Increased rate of major malformations in offspring exposed to valproate during pregnancy. Neurology. 2005; 64(6) 961-5

33. DiLiberti JH, Farndon PA, Dennis NR, Curry CJ. The fetal valproate syndrome. Am J Med Genet. 1984; 19(3): 473-81.

34. Winter RM, Donnai D, Burn J, Tucker SM. Fetal valproate síndrome: is there a recognisable phenotype? J Med Genet. 1987; 24(11): 692-695.

35. Kini U, Adab N, Vinten J Fryer A, Clayuton-Smith J, Group LaMNS. Dysmorfhic features: an important clue to the diagnosis and severity of fetal anticonvulsivant syndromes. Arch Dis Child Feteal Neonatal. 2006; 91(2):F90-95.

36. Holmes LB, Coull BA, Dorfman J, Rosenberger PB. The correlation of deficits in IQ with midface and digit hypoplasia in children exposed in utero to anticonvulsant drugs. The Journal of Pediatrics. 146(1):118-22 doi: 10.1016/j. jpeds.2004.08.048

37. Vinten J, Adab N, Kini U, Gorry J, Gregg J, Baker GA, et al. Neuropsychological effects of exposure to anticonvulsant medication in utero. Neurology. 2005;64(6):949-54. doi: 10.1212/01.WNL.0000154514.82948.69.

38. Tomson T, Battino D. Teratogenic effects of antiepileptic drugs. The Lancet Neurology. 2012;11(9):803-13. doi:10.1016/S1474-4422(12)70103-5.

39. Adab N, Jacoby A, Smith D, Chadwick D. Additional educational needs in children born to mothers with epilepsy. Journal of Neurology, Neurosurgery \& Psychiatry. 2001;70(1):15-21. doi:10.1136/jnnp.70.1.15

40. Christensen J, Grønborg TK, Sørensen MJ, Schendel D, Parner ET, Pedersen $\mathrm{LH}$, et al. Prenatal valproate exposure and risk of autism spectrum disorders and childhood autism. Jama. 2013;309(16):1696-703. doi:10.1001/jama.2013.2270.

41. Díaz-Anzaldúa A, Díaz-Martínez A. Contribución genética, ambiental y epigenética en la susceptibilidad a los trastornos del espectro autista. Rev Neurol. 2013;57(556):68.

42. Reinisch J, Sanders SA, Mortensen E, Rubin DB. In Utero Exposure to Phenobarbital and Intelligence Deficits in Adult Men. JAMA. 1995;274(19):1518-1525. doi:10.1001/jama.1995.03530190032031

43. Beghi E, Annegers JF. Pregnancy registries in epilepsy. Epilepsia 2001;42(11):1422-5.

44. Holmes LB, Wyszynski DF, Lieberman E. The AED (antiepileptic drug) pregnancy registry: a 6-year experience. Archives of Neurology. 2004;61(5):673-8. doi:10.1001/archneur.61.5.673.

45. Gedzelman E, Meador KJ. Antiepileptic drugs in women with epilepsy during pregnancy. Therapeutic advances in drug safety. 2012:2042098611433192. doi: $10.1177 / 2042098611433192$.

46. Wide K, Winbladh B, Källén B. Major malformations in infants exposed to antiepileptic drugs in utero, with emphasis on carbamazepine and valproic acid: a nation-wide, population-based register study. Acta Pædiatrica. 2004;93(2):174-6. doi: 10.1111/j.1651-2227.2004.tb00701.x. 
47. Jentink J, Dolk H, Loane MA, Morris JK, Wellesley D, Garne E, et al. Intrauterine exposure to carbamazepine and specific congenital malformations: systematic review and case-control study2010 2010-12-03 00:09:25. doi. 10.1136/bmj.c6581.

48. Battino D, Tomson T, Bonizzoni E, Craig J, Lindhout D, Sabers A, et al. Seizure control and treatment changes in pregnancy: Observations from the EURAP epilepsy pregnancy registry. Epilepsia. 2013;54(9):1621-7. doi: 10.1111/epi.12302

49. Cunnington M, Ferber S, Quartey G. Effect of dose on the frequency of major birth defects following fetal exposure to lamotrigine monotherapy in an international observational study. Epilepsia. 2007;48(6):1207-10.

50. Artama M, Auvinen A, Raudaskoski T, Isojärvi I, Isojärvi J. Antiepileptic drug use of women with epilepsy and congenital malformations in offspring. Neurology. 2005;64(11):1874-8. doi:10.1212/01.WNL.0000163771.96962.1F.

51. Hernández-Díaz S, Smith C, Shen A, Mittendorf R, Hauser W, Yerby M, et al. Comparative safety of antiepileptic drugs during pregnancy. Neurology. 2012;78(21):1692-9. doi: 10.1212/WNL.0b013e3182574f39.

52. Hunt S, Craig J, Russell A, Guthrie E, Parsons L, Robertson I, et al. Levetiracetam in pregnancy: preliminary experience from the UK Epilepsy and Pregnancy Register. Neurology. 2006;67(10):1876-9. doi:10.1212/01.wnl.0000244491. 48937.55.

53. Mawhinney E, Craig J, Morrow J, Russell A, Smithson WH, Parsons L, et al. Levetiracetam in pregnancy Results from the UK and Ireland epilepsy and pregnancy registers. Neurology. 2013;80(4):400-5. doi: 10.1212/WNL.0b013e31827f0874.

54. Tomson T, Battino D, Craig J, Hernandez Diaz S, Holmes LB, Lindhout D, et al Pregnancy registries: differences, similarities, and possible harmonization. Epilepsia. 2010;51(5):909-15.

55. Hunt S, Russell A, Smithson W, Parsons L, Robertson I, Waddell R, et al. Topiramate in pregnancy Preliminary experience from the UK Epilepsy and Pregnancy Register. Neurology. 2008;71(4):272-6. doi: 10.1212/01.wnl.0000318293.28278. 33

56. Margulis AV, Mitchell AA, Gilboa SM, Werler MM, Mittleman MA, Glynn $\mathrm{RJ}$, et al. Use of topiramate in pregnancy and risk of oral clefts. American journal of obstetrics and gynecology. 2012;207(5):405. e1-e7. doi:10.1016/j. ajog.2012.07.008

57. Krishnamurthy KB. Managing epilepsy during pregnancy: assessing risk and optimizing care. Current treatment options in neurology. 2012;14(4):348-55. doi: 10.1007/s11940-012-0184-7
58. Werler MM, Ahrens KA, Bosco JL, Mitchell AA, Anderka MT, Gilboa SM, et al. Use of antiepileptic medications in pregnancy in relation to risks of birth defects. Annals of epidemiology. 2011;21(11):842-50. doi: doi:10.1016/j.annepidem.2011.08.002

59. Adab N, Kini U, Vinten J, Ayres J, Baker G, Clayton-Smith J, et al. The longer term outcome of children born to mothers with epilepsy. Journal of Neurology, Neurosurgery \& Psychiatry. 2004;75(11):1575-83. doi:10.1136/jnnp.2003.029132.

60. Meador K, Pennell P, Harden C, Gordon J, Tomson T, Kaplan P, et al. Pregnancy registries in epilepsy A consensus statement on health outcomes. Neurology. 2008;71(14):1109-17. doi: 10.1212/01.wnl.0000316199.92256.af.

61. Meador K, Baker G, Browning N, Cohen M, Bromley R, Clayton-Smith J, et al. Effects of fetal antiepileptic drug exposure Outcomes at age 4.5 years. Neurology. 2012;78(16):1207-14. doi: 10.1212/WNL.0b013e318250d824.

62. Meador KJ, Baker GA, Browning N, Cohen MJ, Bromley RL, Clayton-Smith J, et al. Fetal antiepileptic drug exposure and cognitive outcomes at age 6 years (NEAD study): a prospective observational study. The Lancet Neurology. 2013;12(3):244-52. doi:10.1016/S1474-4422(12)70323-X

63. Czeizel AE. Prevention of congenital abnormalities by periconceptional multivitamin supplementation. Bmj. 1993;306(6893):1645-8. doi:10.1136/ bmj.306.6893.1645.

64. Martínez-Frías ML, Rodríguez-Pinilla E, Bermejo E. Análisis de la situación en España sobre el consumo de ácido fólico/folinato cálcico para la prevención de defectos congénitos. Medicina Clínica. 2003;121(20):772-5. doi:10.1016/S00257753(03)74094-9.

65. Kaaja E, Kaaja R, Hiilesmaa V. Major malformations in offspring of women with epilepsy. Neurology. 2003;60(4):575-9. doi:10.1212/01.WNL.0000044157. 28073.DC.

66. Morrow JI, Hunt SJ, Russell AJ, Smithson WH, Parsons L, Robertson I, et al. Folic acid use and major congenital malformations in offspring of women with epilepsy: a prospective study from the UK Epilepsy and Pregnancy Register. Journal of Neurology, Neurosurgery \& Psychiatry. 2009;80(5):506-11. doi:10.1136/ jnnp.2008.156109.

67. Cheschier N. ACOG practice bulletin. Neural tube defects. Number 44, July 2003.(Replaces committee opinion number 252, March 2001). International journal of gynaecology and obstetrics: the official organ of the International Federation of Gynaecology and Obstetrics. 2003;83(1):123-33. doi:10 10.1016/S00207292(03)00390-4.1016/S0020-7292(03)00390-4 